Questionnaire (SGRQ) (Tsiligianni IG et al 2012). Although the CRO and SGRQ were originally developed in patients with chronic airway obstruction, they are commonly used in clinical practise in chronic respiratory diseases other than COPD. We hypothesised that the CCQ would correlate with existing health status measures and exercise capacity in a survey of non-COPD patients

Methods 60 patients were recruited from respiratory outpatient clinics. Disease classifications included interstitial lung disease $(n=23)$, asthma $(n=10)$, bronchiectasis $(n=17)$, extrathoracic restriction $(n=8)$ and thoracic surgery for lung cancer $(n=2)$. CCQ, CAT, CRO, SGRQ and incremental shuttle walk (ISW) were recorded. Spearman's rank correlation was used to assess the relationship between CCQ and other outcome measures.

Results Baseline characteristics are presented as mean (standard deviation) or median $\left(25^{\text {th }}, 75^{\text {th }}\right.$ percentiles); Age $65(58,77), \mathrm{FEV}_{1} \%$ predicted 69.8 (24.4), BMI 28.0 (25.4, 32.3), MRC 3(1), CCO 2.1 $(1.5,3.8)$ and ISW $210(90,320)$. There was a significant correlation between the total CCQ and CAT, SGRQ, CRDQ, MRC and ISW (all $\mathrm{p}<0.01$ see Table. 1). In addition individual domains of the CCO correlated significantly with MRC, CAT and individual domains of the CRQ $(p<0.01)$.

Conclusions The CCQ correlates well with existing health status and functional outcome measures in non-COPD patients. CCQ may be a useful assessment tool to test the efficacy of interventions such as pulmonary rehabilitation in this population, but longitudinal studies are required to confirm.

\section{P107 LONG-TERM ADHERENCE TO EXERCISE AFTER PULMONARY REHABILITATION: UNDERSTANDING THE MOTIVATIONS AND BARRIERS TO EXERCISE?}

doi:10.1136/thoraxjnl-2012-202678.390

C Lee, S Elkin. Imperial College Healthcare NHS Trust, London, UK

Introduction Adherence to exercise after PR is known to be low and the role of motivation/barriers in this population remains unclear. This study aimed to further investigate the role of motivation and barriers to exercise and specifically profiling trends that may guide/assist maintenance strategies.

Method $112(58=\mathrm{M})$ patients who completed $>50 \%$ of a PR programme over a 3 year period, were sent a postal survey. Data collected included; demographics, co-morbidities, MRC, physical activity/exercise and motivation (21 items) and barriers (14 items) to exercise quantified along a 5 point-likert scale (Newson and Kemps, 2007).

Results $51.8 \%(\mathrm{n}=58)$ responded; mean age 71.72 , MRC dyspnoea 2.86 and co-morbidities $1.09, \mathrm{COPD}=87.9 \%$. Motivation and barriers were analysed separately, as mean motivation/barriers weren't significantly correlated. Mean barrier was significant correlated with MRC dyspnoea $(p=0.003)$, co-morbidities $(p<0.001)$ and intent to exercise $(p<0.001)$, but not with motivation. Sub-analyses identified those who exercised regularly (currently at least once a week most weeks) rated motivations and barriers significantly different to those who didn't. Exercisers had significantly higher mean motivation $(p=0.023)$ and deemed the following factors to be significantly more motivating reasons to exercise; 'I want to be physically fit' ( $p=0.002)$; 'I exercise because a health professional advised me to' $(p=0.029)$; 'I want to stay in shape' $(p=0.019)$ and 'exercising gives me energy' $(p=0.0210)$. Conversely the non-exercisers had significantly higher mean barrier score $(p=0.003)$ and rated the following as significantly greater barriers; 'Shortness of breath' ( $p=0.013)$; 'lack of energy' $(p=0.011)$; 'having a limited health/physical condition' ( $p=0.028)$; 'painful joints' $(p=0.002)$; 'not knowing what you are capable of, or should be doing' $(p=0.030)$; fear of, injury $(p=0.032)$, falling $(p=0.036)$ and safety $(p=0.021)$. None of these factors changed over time since completing PR.

Conclusion This study quantified 21 motivating factors to exercise and 14 barriers that prevent exercise in the post-PR population. Some factors were rated significantly differently between exercisers and non-exercisers and did not significantly vary over time since completion of PR. Further research is required to establish if targeting specific factors could guide/assist maintenance strategies.

\section{P108 HEALTHCARE PROFESSIONALS PERCEPTIONS OF SELF MANAGEMENT IN COPD - IMPORTANT, CHALLENGING AND MISUNDERSTOOD}

doi:10.1136/thoraxjnl-2012-202678.391

HML Young, S Harrison, LD Apps, VL Warrington, SJ Singh. University Hospitals of Leicester NHS Trust, Leicester, UK

Introduction There is increasing focus on the importance of self management (SM) within COPD. Literature from other chronic diseases highlights the challenges of implementing SM and the lack of specialist training available. Currently we have no knowledge of what Health Care Professionals (HCP) understand by the term 'SM' and their perceptions of the challenges to successfully supporting SM strategies specifically with a COPD population. A greater awareness of HCP understanding and beliefs surrounding SM is likely to inform training, enhance professional development and improve delivery of SM to patients with COPD.

Aim To explore HCP understanding of SM and their perceptions of the challenges of supporting COPD patients with SM in order to identify education, training and resource needs.

Method A purposive sample of 14 respiratory HCP participated in semi-structured interviews. Interviews were transcribed verbatim and thematic analysis was performed. Two experienced researchers ( $\mathrm{SH}, \mathrm{LA}$ ) analysed a sub-group of interviews to enhance rigour.

Results Three main themes emerged from the data: 1. Understanding of $S M$ - meaning, importance. 2. Supporting $S M$ - advising and working in partnership. 3. Challenges to delivering $S M$ - service, cultural and perceived patient barriers.

Conclusion HCP demonstrate a lack of understanding regarding SM which subsequently impacts upon its successful delivery. In spite of this, all HCP agreed that SM is an important aspect of care for COPD patients. HCP feel apt in the delivery of advice and many worked in partnership with their patients but most failed to consistently address additional SM needs. HCP could improve their delivery of individualised SM by abandoning preconceptions about their patients and enhancing skills to assist patients with core SM strategies including: problem solving, decision making and taking action.

Abstract P106 Table 1 Relationship between CCO and other outcome measures, $r=$ Spearman's Correlation Coefficient

\begin{tabular}{lccccccccccc}
\hline & ISW $(\mathbf{m})$ & MRC & CAT & CRQ-D & CRQ- & CRO-E & CRO-M & SGRO Symptom & SGRO Activities & SGRO Impact & SGRO Total \\
\hline CCO Symptoms & -0.41 & 0.54 & 0.63 & -0.46 & -0.42 & -0.50 & -0.58 & 0.47 & 0.61 & 0.55 & 0.63 \\
CCO Functional & -0.44 & 0.52 & 0.65 & -0.42 & -0.67 & -0.78 & -0.72 & 0.21 & 0.58 & 0.75 & 0.66 \\
CCO Mental & -0.59 & 0.64 & 0.67 & -0.64 & -0.63 & -0.62 & -0.76 & 0.27 & 0.81 & 0.71 & 0.77 \\
CCO Total & -0.54 & 0.65 & 0.74 & -0.59 & -0.66 & -0.71 & -0.79 & 0.37 & 0.78 & 0.76 & 0.79 \\
\hline
\end{tabular}


Addressing service challenges such as lack of time, prioritisation of achieving QOF indicators and increasing workload demands, as well as the cultural challenges created by professional hierarchies may allow HCP to deliver quality SM more consistently and effectively.

\section{Severe lung disease progression and transplantation}

\section{P109 CIRCULATING MMP ACTIVITY AND LUNG REMODELLING IN LAM}

doi:10.1136/thoraxjnl-2012-202678.392

${ }^{1} \mathrm{JL}$ Cane, ${ }^{1} \mathrm{~W} Y$ Chang, ${ }^{2} \mathrm{R}$ Gallagher, ${ }^{2} \mathrm{~V}$ Gontu, ${ }^{2} \mathrm{M}$ Kumaran, ${ }^{1} \mathrm{SR}$ Johnson. 'Division of Therapeutics and Molecular Medicine, The University of Nottingham, Nottingham, United Kingdom; ${ }^{2}$ Department of Radiology, University Hospitals NHS Trust, Nottingham, United Kingdom

LAM is characterised by the progressive accumulation of lung cysts. It is possible increased proteolysis causes extra-cellular matrix breakdown leading to cyst formation. Matrix metalloproteinases (MMPs) are expressed in the lungs and serum of patients with LAM and can break down the extracellular matrix. Here we examined MMP expression and activity patients with LAM and healthy women and related MMP activity to extent and activity of lung disease.

59 patients with LAM and 32 healthy controls were recruited. Ethical approval was obtained and all gave informed consent. Serum was collected in separator tubes and processed within 30 minutes, urine was centrifuged at $4^{\circ} \mathrm{C}$ and all samples were stored in aliquots at $-80^{\circ} \mathrm{C}$. MMP- 2 and -9 were measured by ELISA and gelatin zymography. Urine results were normalised against creatinine concentration prior to analysis. Lung function data was obtained from clinical records. Cyst volume was measured on a Philips MX8000 IDT 16 slice spiral CT scanner using density mask software on a Philips Healthcare Q19.5 Extended Brilliance ${ }^{\mathrm{TM}}$ Workspace. The trachea and large airways were excluded, cysts were defined as having a threshold density of $<-900$ Hounsfield units and cyst volume expressed as a percentage of total lung volume. Rate of decline for $\mathrm{FEV}_{1}$ was estimated from symptom detection to current lung function. Data were analysed using non parametric Mann-Whitney U tests and linear regression.

Total serum MMP-2 ( $p<0.01)$, total MMP-9 $(p<0.001)$ and active MMP-9 $(p<0.05)$ assessed by zymography were greater in patients than controls. Urine MMP-9 did not differ. Total serum MMP-2 was associated with preserved FEV $\left(p<0.01, r^{2}=0.040\right)$. Cyst volume was correlated with reduction in $\mathrm{FEV}_{1}$ and $\mathrm{FEV}_{1} / \mathrm{FVC}$ ratio but not with any MMP measurement. Median FEV decline was $108.5 \mathrm{ml} /$ year (range 0-840): no measurement of MMP expression or activity was correlated with rate of decline.

Total and active MMP-9 were raised in serum from LAM patients but were not associated with clinical course. Surprisingly, higher serum MMP-2 was associated with preserved lung function. It is not clear if circulating MMP levels reflect the situation in the lung and further analysis of MMPs as a therapeutic target for LAM is required.

\section{P110 INTERLEUKIN-33 IN CHRONIC LUNG DISEASE}

doi:10.1136/thoraxjnl-2012-202678.393

NJ Green, MI Suwara, LA Borthwick, DA Mann, AJ Fisher. Newcastle University, Newcastle Upon Tyne, UK,

Background IL33, a member of the IL-1 superfamily has been implicated in the pathogenesis of asthma and postulated to play an aetiological role in several non-pulmonary fibrotic diseases. IL-33 expression has predominantly been reported in mucosal surfaces. We hypothesised lung epithelium might act as a source of IL33 release into the microenvironment as a damage associated molecular pattern (DAMP); propagating pro-inflammatory/fibrotic pathways.

We evaluated tissue expression of IL-33 from a range of chronic lung diseases and assessed release in response to airway epithelial damage in vitro. Finally, we determined if IL-33 was detectable in BAL of lung transplant patients developing Bronchiolitis Obliterans Syndrome (BOS).

Methods Expression of IL-33 in chronic lung disease was evaluated by immunohistochemistry from patients with $\operatorname{IPF}(n=3)$, $\operatorname{COPD}(n=3)$, Bronchiectasis $(n=3)$ and $\operatorname{CF}(n=3)$. Epithelial damage was induced in Primary Bronchial Epithelial Cells and 16HBE14 cells by oxidative stress or freeze/thaw and release of IL33 evaluated by ELISA and Western Blot. BAL was prospectively collected $(n=207)$ from post lung transplant patients $(\mathrm{n}=26)$ and IL-33 concentration measured by ELISA. BAL samples were classified as Non $\operatorname{BOS}(n=116)$ or $\operatorname{BOS}(n=91)$ on the basis of histological and clinical data. Co-existing presence of infection was identified by standard microbiological culture.

Results IL33 was strongly expressed in airway epithelia with a predominant nuclear location. This was more marked in chronic lung diseases with an infective aetiology (CF and bronchiectasis). IL33 was not detectable in response to airway epithelial cell injury in vitro. However, IL-33 levels were elevated in BAL of individuals with $\operatorname{BOS}(p=0.011)$. Longitudinal analysis of 26 individuals spanning the time frame of initial BOS diagnosis demonstrated a trend towards increased concentration of IL-33 in BAL in the immediate period following BOS diagnosis. There was a strong association between elevated IL-33 levels and the presence of $\mathrm{BOS}$ with concomitant infection $(p<0.001)$

Conclusion IL33 is strongly expressed in the airway epithelium in chronic respiratory diseases but does not appear to be passively released as a DAMP in airway epithelial cell damage. Elevated IL33 with infection in the post transplant population suggests sources other than epithelium may be important but further work is required to evaluate the relevance and significance of these observations.

\section{P111 BRONCHOALVEOLAR LAVAGE DOES NOT AFFECT THE ACUTE INFLAMIMATORY RESPONSE FOLLOWING BRONCHOSCOPY AND MEDIASTINOSCOPY}

doi:10.1136/thoraxjnl-2012-202678.394

${ }^{1}$ RO Jones, $2 \mathrm{JT}$ Murchison, ${ }^{2} \mathrm{G}$ Casali, ${ }^{2} \mathrm{EJ}$ Simon, ${ }^{3} \mathrm{~N}$ Anderson, ${ }^{4} \mathrm{AJ}$ Simpson, ${ }^{2}$ WS Walker. 'The Queen's Medical Research Institute, The University of Edinburgh, Edinburgh, United Kingdom; ${ }^{2}$ The Royal Infirmary of Edinburgh, Edinburgh, UK; ${ }^{3}$ The University of Edinburgh, Edinburgh, UK; ${ }^{4}$ The University of Newcastle, Newcastle, UK

Objective Bronchoalveolar lavage (BAL) may be used to investigate acute inflammation following thoracic surgery. However, BAL has previously been found to induce an acute phase response in healthy and critically-ill patients complicating research analysis $(1,2)$. We sought to examine the impact of BAL in thoracic surgery patients hypothesising that BAL would not lead to a significant additional acute inflammatory response.

Methods Seventeen patients undergoing lung cancer staging bronchoscopy and mediastinoscopy were randomly assigned to have $220 \mathrm{mls} 0.9 \% \mathrm{NaCl}$ BAL before surgery $(n=10)$ or no BAL $(n=7)$. Blood samples were taken pre-operatively followed by 6 and 24 hours post-operatively. Exhaled nitric oxide (eNO) was also measured at a flow rate of $50 \mathrm{mls} / \mathrm{sec}$ at these times. All patients had a CXR at $24 \mathrm{hrs}$ and were evaluated for evidence of SIRS using predefined criteria. 